\title{
Further Application of $H$-Differentiability to Generalized Complementarity Problems Based on Generalized Fisher-Burmeister Functions
}

\author{
Wei-Zhe Gu ${ }^{1,2}$ and Mohamed A. Tawhid ${ }^{2,3}$ \\ ${ }^{1}$ Department of Mathematics, School of Science, Tianjin University, Tianjin 300072, China \\ ${ }^{2}$ Department of Mathematics and Statistics, Faculty of Science, Thompson Rivers University, Kamloops, BC, Canada V2C 0C8 \\ ${ }^{3}$ Department of Mathematics and Computer Science, Faculty of Science, Alexandria University, Moharram Bey, \\ Alexandria 21511, Egypt
}

Correspondence should be addressed to Mohamed A. Tawhid; mtawhid@tru.ca

Received 16 July 2014; Accepted 28 August 2014; Published 17 November 2014

Academic Editor: Janusz Brzdek

Copyright (c) 2014 W.-Z. Gu and M. A. Tawhid. This is an open access article distributed under the Creative Commons Attribution License, which permits unrestricted use, distribution, and reproduction in any medium, provided the original work is properly cited.

We study nonsmooth generalized complementarity problems based on the generalized Fisher-Burmeister function and its generalizations, denoted by $\operatorname{GCP}(f, g)$ where $f$ and $g$ are $H$-differentiable. We describe $H$-differentials of some GCP functions based on the generalized Fisher-Burmeister function and its generalizations, and their merit functions. Under appropriate conditions on the $H$-differentials of $f$ and $g$, we show that a local/global minimum of a merit function (or a "stationary point" of a merit function) is coincident with the solution of the given generalized complementarity problem. When specializing $\mathrm{GCP}(f, g)$ to the nonlinear complementarity problems, our results not only give new results but also extend/unify various similar results proved for $C^{1}$, semismooth, and locally Lipschitzian.

\section{Introduction}

Gowda et al. in [1] introduced the concepts of the $H$ differentiability and $H$-differential for a function $f: R^{n} \rightarrow$ $R^{n}$. They showed that the Fréchet derivative of a Fréchet differentiable function, the Clarke generalized Jacobian of a locally Lipschitzian function [2], the Bouligand subdifferential of a semismooth function [3-5], and the $C$ differential of a $C$-differentiable function [6] are instances of $H$-differentials. In their paper, they noted that $\mathrm{H}$ differentials enjoy simple sum, product, and chain rules, $H$ differentiability implies continuity, and any superset of an $\mathrm{H}$ differential is an $\mathrm{H}$-differential. It is noted in [7] that the $\mathrm{H}$ differentiable function needs not be locally Lipschitzian nor directionally differentiable.

There have been many applications of these concepts to optimization, complementarity problems, and variational inequalities, characterizations of $\mathbf{P}\left(\mathbf{P}_{\mathbf{0}}\right)$ and $\mathbf{E}\left(\mathbf{E}_{\mathbf{0}}\right)$ properties when the underlying functions are not necessarily locally Lipschitzian or semismooth (see, e.g., [7-15]).
In this paper, we study a further application of $H$ differentiability to nonsmooth generalized complementarity problems based on the generalized Fisher-Burmeister function and its generalizations, denoted by $\operatorname{GCP}(f, g)$ where $f$ and $g$ are $H$-differentiable.

We consider a generalized complementarity problem corresponding to $H$-differentiable functions $f$ and $g$, denoted by $\operatorname{GCP}(f, g)$, which is to find a vector $x^{*} \in \mathfrak{R}^{n}$ such that

$$
f\left(x^{*}\right) \geq 0, \quad g\left(x^{*}\right) \geq 0, \quad\left\langle f\left(x^{*}\right), g\left(x^{*}\right)\right\rangle=0,
$$

where $f: \mathfrak{R}^{n} \rightarrow \mathfrak{R}^{n}$ and $g: \mathfrak{R}^{n} \rightarrow \mathfrak{R}^{n}$.

In the last decades, many researchers have given a lot of attention to this problems in terms of its applications, numerical methods, and formulation; see $[16,17]$ and the references cited therein. If $g(x)=x-T(x)$ with some $T$ : $R^{n} \rightarrow R^{n}$, then $\operatorname{GCP}(f, g)$ is known as the quasi/implicit complementarity problem; see, for example, [17-19]. Also, if $g(x)=x$, then $\operatorname{GCP}(f, g)$ reduces to the nonlinear complementarity problem $\mathrm{NCP}(f)$. By taking in $\operatorname{NCP}(f)$ 
$f(x)=M x+q$ with $M \in R^{n \times n}$ and a vector $q \in R^{n}$, then $\operatorname{NCP}(f)$ is called a linear complementarity problem $\operatorname{LCP}(M, q)$.

Our approach is to reformulate $\operatorname{GCP}(f, g)$ as an unconstrained optimization problem through some merit function. We construct a merit function via a GCP function $\phi: R^{2} \rightarrow$ $R$ :

$$
\phi(a, b)=0 \Longleftrightarrow a b=0, \quad a \geq 0, b \geq 0 .
$$

For the problem $\operatorname{GCP}(f, g)$, we define

$$
\begin{gathered}
\Phi(x)=\left[\phi\left(f_{1}(x), g_{1}(x)\right), \ldots, \phi\left(f_{i}(x), g_{i}(x)\right), \ldots,\right. \\
\left.\phi\left(f_{n}(x), g_{n}(x)\right)\right]^{T}
\end{gathered}
$$

and we call $\Phi(x)$ a GCP function for $\operatorname{GCP}(f, g)$. A function $\Psi: R^{n} \rightarrow[0, \infty)$ is said to be a merit function for $\operatorname{GCP}(f, g)$ provided that the global minima of $\Psi$ are coincident with the solutions of the original $\operatorname{GCP}(f, g)$. We consider a GCP function $\Phi: R^{n} \rightarrow R^{n}$ associated with $\operatorname{GCP}(f, g)$ and its merit function

$$
\Psi(x):=\frac{1}{2}\|\Phi(x)\|^{2}
$$

so that

$$
\bar{x} \text { solves } \operatorname{GCP}(f, g) \Longleftrightarrow \Phi(\bar{x})=0 \Longleftrightarrow \Psi(\bar{x})=0 \text {. }
$$

The organization of the paper is as follows. We state some basic definitions and preliminary results. We describe $H$ differentials of some GCP functions based on the generalized Fisher-Burmeister function and its generalizations and their merit functions. We show that under appropriate show $\mathbf{P}_{\mathbf{0}}(\mathbf{P})$ conditions and column $\mathbf{P}$ property conditions, local/global minimum of a merit function (or a "stationary point" or "semi-stationary point" of a merit function) based on the generalized Fisher-Burmeister function and its generalizations coincides with the solution of the given generalized complementarity problem. Note that considering GCP functions on the basis of the generalized Fisher-Burmeister function and its generalizations seems to be new.

Moreover, when specializing GCP $(f, g)$ to the nonlinear complementarity problems, our results not only give new results but also extend/unify various similar results proved for $C^{1}$, semismooth, and locally Lipschitzian.

\section{Preliminaries}

Throughout this paper, all vectors in $R^{n}$ are column vectors. $x^{T} y$ or $\langle x, y\rangle$ denotes the inner product between two vectors $x$ and $y$ in $R^{n}$. Vector inequalities are interpreted componentwise. All the operations are performed componentwise. For a set $E \subseteq R^{n}$, co $E$ denotes the convex hull of $E$ and $\overline{\mathrm{co}} E$ denotes the closure of co $E$. For a differentiable function $f: R^{n} \rightarrow R^{m}, \nabla f(\bar{x})$ denotes the Jacobian matrix of $f$ at $\bar{x}$. For a matrix $A, A_{i}$ denotes the $i$ th row of $A$. $\|x\|_{p}$ denotes the $p$-norm of $x$ and $\|x\|$ denotes the Euclidean norm of $x$. In addition, unless otherwise stated, assume $p$ in the sequel is any fixed real number in $(1, \infty)$.

In this section, we first recall some background concepts.
We first recall the definition of $H$-differentiability and examples from [1].

Definition 1. Given a function $F: \Omega \subseteq \mathfrak{R}^{n} \rightarrow \mathfrak{R}^{m}$, where $\Omega$ is an open set in $\mathfrak{R}^{n}$ and $x^{*} \in \Omega$, we say that a nonempty subset $T\left(x^{*}\right)$, also denoted by $T_{F}\left(x^{*}\right)$, of $\Re^{m \times n}$ is an $H$ differential of $F$ at $x^{*}$ if for every sequence $x^{k} \in \Omega$ converging to $x^{*}$, there exist a subsequence $x^{k_{j}}$ and a matrix $A \in T\left(x^{*}\right)$ such that

$$
F\left(x^{k_{j}}\right)-F\left(x^{*}\right)-A\left(x^{k_{j}}-x^{*}\right)=o\left(\left\|x^{k_{j}}-x^{*}\right\|\right) .
$$

We say that $F$ is $H$-differentiable at $x^{*}$ if $F$ has an $H$ differential at $x^{*}$.

A useful equivalent definition of an $H$-differential $T_{F}\left(x^{*}\right)$ is as follows: for any sequence $x^{k}:=x^{*}+t_{k} d^{k}$ with $t_{k} \downarrow 0$ and $\left\|d^{k}\right\|=1$ for all $k$, there exist convergent subsequences $t_{k_{j}} \downarrow 0$ and $d^{k_{j}} \rightarrow d$, and $A \in T_{F}\left(x^{*}\right)$ such that

$$
\lim _{j \rightarrow \infty} \frac{F\left(x^{*}+t_{k_{j}} d^{k_{j}}\right)-F\left(x^{*}\right)}{t_{k_{j}}}=A d .
$$

Here are some well-known facts about $H$-differentiability; see, for example, $[8-10,15]$.

Remark 2. (i) Any superset of an $H$-differential is an $H$ differential.

(ii) $H$-differentiability implies continuity, and $H$ differentials enjoy simple sum, product, and chain rules.

(iii) While the Fréchet derivative of a differentiable function, the Clarke generalized Jacobian of a locally Lipschitzian function [2], the Bouligand differential of a semismooth function [4], and the $C$-differential of a $C$-differentiable function [6] are particular instances of $H$-differential, it is shown in [10] by example that an $H$-differentiable function need not be locally Lipschitzian nor directionally differentiable.

(iv) If a function $F: \Omega \subseteq R^{n} \rightarrow R^{m}$ is $H$-differentiable at a point $\bar{x}$, then there exist a constant $L>0$ and a neighbor$\operatorname{hood} B(\bar{x}, \delta)$ of $\bar{x}$ with

$$
\|F(x)-F(\bar{x})\| \leq\|x-\bar{x}\| \quad \forall x \in B(\bar{x}, \delta) .
$$

Conversely, if condition (8) holds, then $T(\bar{x}):=\mathfrak{R}^{m \times n}$ can be taken as an $H$-differential of $F$ at $\bar{x}$.

In [10], the following definition is introduced to generalize the concepts of monotonicity, $\mathbf{P}_{\mathbf{0}}$-property, and their variants for function in [20].

Definition 3. For functions $f, g: \mathfrak{R}^{n} \rightarrow \mathfrak{R}^{n}$, we say that $f$ and $g$ are as follows:

(a) relatively monotone if

$$
\langle f(x)-f(y), g(x)-g(y)\rangle \geq 0 \quad \forall x, y \in \mathfrak{R}^{n},
$$

(b) relatively strictly monotone if

$$
\langle f(x)-f(y), g(x)-g(y)\rangle>0 \quad \forall x, y \in \mathfrak{R}^{n},
$$


(c) relatively strongly monotone if there exists a constant $\mu>0$ such that

$\langle f(x)-f(y), g(x)-g(y)\rangle \geq \mu\|x-y\|^{2} \quad \forall x, y \in \mathfrak{R}^{n}$,

(d) relatively $\mathbf{P}_{\mathbf{0}}(\mathbf{P})$-functions if for any $x \neq y$ in $\mathfrak{R}^{n}$,

$$
\max _{i: x_{i} \neq y_{i}}[f(x)-f(y)]_{i}[g(x)-g(y)]_{i} \geq(>) 0,
$$

(e) relatively uniform $(\mathbf{P})$-functions if there exists a constant $\eta>0$ such that for any $x, y \in \mathfrak{R}^{n}$,

$$
\max _{1 \leq i \leq n}[f(x)-f(y)]_{i}[g(x)-g(y)]_{i} \geq \eta\|x-y\|^{2} .
$$

\section{H-Differentials of Some GCP Functions When the Underlying Functions Are $H$-Differentiable}

In this section, we compute the $H$-differentials of some GCP functions and their merit functions.

Example 4. Suppose that $f$ and $g$ are $H$-differentiable at $\bar{x}$ with $H$-differentials, respectively, by $T_{f}(\bar{x})$ and $T_{g}(\bar{x})$. Consider the following GCP function which is the basis of

$$
\phi_{p}(a, b):=a+b-\|(a, b)\|_{p}
$$

where $p$ is any fixed real number in the interval $(1,+\infty)$ and $\|(a, b)\|_{p}$ denotes the $p$-norm of $(a, b)$; that is, $\|(a, b)\|_{p}=$ $\sqrt[p]{|a|^{p}+|b|^{p}}$. The function $\phi_{p}$ was noted by Tseng [21]. For further study on this family of NCP functions, see [22]. The $i$ th component of this kind of GCP function $\Phi_{p}(x)$ in (3) is defined as

$$
\begin{aligned}
\left(\Phi_{p}(x)\right)_{i} & =\phi_{p}\left(f_{i}(x), g_{i}(x)\right) \\
& :=f_{i}(x)+g_{i}(x)-\left\|\left(f_{i}(x), g_{i}(x)\right)\right\|_{p} .
\end{aligned}
$$

Now we describe the $H$-differentials of $\Phi_{p}$. Let

$$
J(\bar{x}):=\left\{i: f_{i}(\bar{x})=0=g_{i}(\bar{x})\right\}
$$

The $H$-differential of $\Phi_{p}$ at $\bar{x}$ is given by

$$
T_{\Phi_{p}}(\bar{x})=\{V A+W B:(A, B, V, W, d) \in \Gamma\},
$$

where $\Gamma$ is the set of all quadruples $(A, B, V, W, d)$ with $A \in$ $T_{f}(\bar{x}), B \in T_{g}(\bar{x}),\|d\|=1, V=\operatorname{diag}\left(v_{i}\right)$, and $W=\operatorname{diag}\left(w_{i}\right)$ being diagonal matrices satisfying the conditions:

$$
\begin{aligned}
& \left|1-v_{i}\right|^{p /(p-1)}+\left|1-w_{i}\right|^{p /(p-1)}=1, \quad \forall i=1,2, \ldots, n, \\
& \int 1-\frac{\left|f_{i}(\bar{x})\right|^{p-1} \operatorname{sgn}\left(f_{i}(x)\right)}{\left(\left|f_{i}(\bar{x})\right|^{p}+\left|g_{i}(\bar{x})\right|^{p}\right)^{(p-1) / p}}, \\
& i \notin J(\bar{x}) \text {, } \\
& v_{i}=\left\{1-\frac{\left|A_{i} d\right|^{p-1} \operatorname{sgn}\left(A_{i} d\right)}{\left(\left|A_{i} d\right|^{p}+\left|B_{i} d\right|^{p}\right)^{(p-1) / p}},\right. \\
& i \in J(\bar{x}), \quad\left|A_{i} d\right|^{p}+\left|B_{i} d\right|^{p}>0, \\
& \text { arbitrary, } \\
& i \in J(\bar{x}), \quad\left|A_{i} d\right|^{p}+\left|B_{i} d\right|^{p}=0, \\
& w_{i}=\left\{\begin{array}{l}
1-\frac{\left|g_{i}(\bar{x})\right|^{p-1} \operatorname{sgn}\left(g_{i}(\bar{x})\right)}{\left(\left|f_{i}(\bar{x})\right|^{p}+\left|g_{i}(\bar{x})\right|^{p}\right)^{(p-1) / p},} \\
\quad i \notin J(\bar{x}), \\
1-\frac{\left|B_{i} d\right|^{p-1} \operatorname{sgn}\left(B_{i} d\right)}{\left(\left|A_{i} d\right|^{p}+\left|B_{i} d\right|^{p}\right)^{(p-1) / p},} \\
\quad i \in J(\bar{x}), \quad\left|A_{i} d\right|^{p}+\left|B_{i} d\right|^{p}>0, \\
\operatorname{arbitrary,} \\
\quad i \in J(\bar{x}),\left|A_{i} d\right|^{p}+\left|B_{i} d\right|^{p}=0 .
\end{array}\right.
\end{aligned}
$$

Proof. To see this claim, let $\bar{x}+t_{k} d^{k} \rightarrow \bar{x}$ with $t_{k} \downarrow 0$ and $\left\|d^{k}\right\|=1$. By the $H$-differentiability of $f$, there exists a sequence $t_{k_{j}}$ of $t_{k}, d^{k_{j}} \rightarrow d$, and $A \in T_{f}(\bar{x})$, such that

$$
f\left(\bar{x}+t_{k_{j}} d^{k_{j}}\right)-f(\bar{x})-A\left(t_{k_{j}} d^{k_{j}}\right)=o\left(t_{k_{j}}\right) .
$$

Let $y^{k_{j}}:=\bar{x}+t_{k_{j}} d^{k_{j}}$. With $A, B, d, V$, and $W$ satisfying (19), let $C:=V A+W B$. We claim that $\Phi_{p}\left(y^{k_{j}}\right)-\Phi_{p}(\bar{x})-C\left(t_{k_{j}} d^{k_{j}}\right)=$ $o\left(t_{k_{j}}\right)$. To see this, we fix an index $i$ and show that $\left(\Phi_{p}\left(y^{k_{j}}\right)\right)_{i}-$ $\left(\Phi_{p}(\bar{x})\right)_{i}-\left[C\left(t_{k_{j}} d^{k_{j}}\right)\right]_{i}=o\left(t_{k_{j}}\right)$. Without loss of generality, let $i=1$. We denote the 1st row of $A, B$, and $C$ by $A_{1}, B_{1}$, and $C_{1}$. We consider two cases.

Case $1(1 \notin J(\bar{x}))$. In this case, we have

$$
\begin{aligned}
C_{1}= & \left\{1-\frac{\left|f_{i}(\bar{x})\right|^{p-1} \operatorname{sgn}\left(f_{i}(x)\right)}{\left(\left|f_{i}(\bar{x})\right|^{p}+\left|g_{i}(\bar{x})\right|^{p}\right)^{(p-1) / p}}\right\} A_{1} \\
& +\left\{1-\frac{\left|g_{1}(\bar{x})\right|^{p-1} \operatorname{sgn}\left(g_{1}(\bar{x})\right)}{\left(\left|f_{1}(\bar{x})\right|^{p}+\left|g_{1}(\bar{x})\right|^{p}\right)^{(p-1) / p}}\right\} B_{1}, \\
& \left(\Phi_{p}\left(y^{k_{j}}\right)\right)_{1}-\left(\Phi_{p}(\bar{x})\right)_{1}-C_{1} t_{k_{j}} d^{k_{j}}
\end{aligned}
$$




$$
\begin{aligned}
= & A_{1} t_{k_{j}} d^{k_{j}}+B_{1} t_{k_{j}} d^{k_{j}}+o\left(t_{k_{j}}\right) \\
& -\|\left(f_{1}(\bar{x})+A_{1} t_{k_{j}} d^{k_{j}}+o\left(t_{k_{j}}\right),\right. \\
& \left.+g_{1}(\bar{x})+B_{1} t_{k_{j}} d^{k_{j}}+o\left(t_{k_{j}}\right)\right) \|_{p} \\
& -\left\{\left(f_{1}(\bar{x}), g_{1}(\bar{x})\right) \|_{p}\right. \\
& -\left\{1-\frac{\left|f_{1}(\bar{x})\right|^{p-1} \operatorname{sgn}\left(f_{1}(x)\right)}{\left(\left|f_{1}(\bar{x})\right|^{p}+\left|g_{1}(\bar{x})\right|^{p}\right)^{(p-1) / p}}\right\} A_{1} t_{k_{j}} d^{k_{j}} \\
= & o\left(t_{k_{j}}\right) .
\end{aligned}
$$

Case $2(1 \in J(\bar{x}))$

Subcase (1) $\left(\left|A_{1} d\right|^{p}+\left|B_{1} d\right|^{p}>0\right)$. In this case,

$$
\begin{aligned}
& C_{1}=\left\{1-\frac{\left|A_{1} d\right|^{p-1} \operatorname{sgn}\left(A_{1} d\right)}{\left(\left|A_{1} d\right|^{p}+\left|B_{1} d\right|^{p}\right)^{(p-1) / p}}\right\} A_{1} \\
& +\left\{1-\frac{\left|B_{i} d\right|^{p-1} \operatorname{sgn}\left(B_{i} d\right)}{\left(\left|A_{i} d\right|^{p}+\left|B_{i} d\right|^{p}\right)^{(p-1) / p}}\right\} B_{1}, \\
& \left(\Phi_{p}\left(y^{k_{j}}\right)\right)_{1}-\left(\Phi_{p}(\bar{x})\right)_{1}-C_{1} t_{k_{j}} d^{k_{j}} \\
& =A_{1} t_{k_{j}} d^{k_{j}}+B_{1} t_{k_{j}} d^{k_{j}}+o\left(t_{k_{j}}\right) \\
& -\left\|\left(A_{1} t_{k_{j}} d^{k_{j}}+o\left(t_{k_{j}}\right), B_{1} t_{k_{j}} d^{k_{j}}+o\left(t_{k_{j}}\right)\right)\right\|_{p} \\
& -\left\{1-\frac{\left|A_{1} d\right|^{p-1} \operatorname{sgn}\left(A_{1} d\right)}{\left(\left|A_{1} d\right|^{p}+\left|B_{1} d\right|^{p}\right)^{(p-1) / p}}\right\} A_{1} t_{k_{j}} d^{k_{j}} \\
& -\left\{1-\frac{\left|B_{i} d\right|^{p-1} \operatorname{sgn}\left(B_{i} d\right)}{\left(\left|A_{i} d\right|^{p}+\left|B_{i} d\right|^{p}\right)^{(p-1) / p}}\right\} B_{1} t_{k_{j}} d^{k_{j}} \\
& =\left(\frac{\left|A_{1} d\right|^{p-1} \operatorname{sgn}\left(A_{1} d\right)}{\left(\left|A_{1} d\right|^{p}+\left|B_{1} d\right|^{p}\right)^{(p-1) / p}} A_{1} d^{k_{j}}\right. \\
& +\frac{\left|B_{i} d\right|^{p-1} \operatorname{sgn}\left(B_{i} d\right)}{\left(\left|A_{i} d\right|^{p}+\left|B_{i} d\right|^{p}\right)^{(p-1) / p}} B_{1} d^{k_{j}}
\end{aligned}
$$

$$
\begin{aligned}
& \left.\quad-\left\|\left(A_{1} d^{k_{j}}+\frac{o\left(t_{k_{j}}\right)}{t_{k_{j}}}, B_{1} d^{k_{j}}+\frac{o\left(t_{k_{j}}\right)}{t_{k_{j}}}\right)\right\|_{p}\right) t_{k_{j}} \\
& +o\left(t_{k_{j}}\right) \\
& =o\left(t_{k_{j}}\right) .
\end{aligned}
$$

Subcase (2) $\left(\left|A_{1} d\right|^{p}+\left|B_{1} d\right|^{p}=0\right)$. In this case, $A_{1} d=0=$ $B_{1} d$. Then

$$
\begin{aligned}
& \left(\Phi_{p}\left(y^{k_{j}}\right)\right)_{1}-\left(\Phi_{p}(\bar{x})\right)_{1} \\
& =A_{1} t_{k_{j}} d^{k_{j}}+B_{1} t_{k_{j}} d^{k_{j}}+o\left(t_{k_{j}}\right) \\
& \quad-\left\|\left(A_{1} t_{k_{j}} d^{k_{j}}+o\left(t_{k_{j}}\right), B_{1} t_{k_{j}} d^{k_{j}}+o\left(t_{k_{j}}\right)\right)\right\|_{p} \\
& =\left(A_{1} d^{k_{j}}+B_{1} d^{k_{j}}\right. \\
& \left.\quad-\left\|\left(A_{1} d^{k_{j}}+\frac{o\left(t_{k_{j}}\right)}{t_{k_{j}}}, B_{1} d^{k_{j}}+\frac{o\left(t_{k_{j}}\right)}{t_{k_{j}}}\right)\right\|_{p}\right) \\
& \quad \times t_{k_{j}}+o\left(t_{k_{j}}\right) \\
& =o\left(t_{k_{j}}\right) .
\end{aligned}
$$

Example 5. Consider the following GCP function which is based on proposed family of NCP functions [22] relying on $\phi_{p}$ in (14) and some introduced NCP functions in [23]:

$$
\phi_{1}(a, b):=\phi_{p}(a, b)+\alpha a_{+} b_{+}, \quad \alpha>0,
$$

where $a_{+}=\max \{0, a\}$ and GCP function $\Phi(x)$ in (3) is defined as

$$
\Phi_{1}(x):=\phi_{p}(f(x), g(x))+\alpha f(x)_{+} g(x)_{+}, \quad \alpha>0,
$$

where all the operations are performed componentwise.

Let

$$
\begin{gathered}
J(\bar{x}):=\left\{i: f_{i}(\bar{x})=0=g_{i}(\bar{x})\right\}, \\
K(\bar{x}):=\left\{i: f_{i}(\bar{x})>0, g_{i}(\bar{x})>0\right\} .
\end{gathered}
$$

Then the $H$-differential of $\Phi_{1}$ at $\bar{x}$ is given by

$$
T_{\Phi_{1}}(\bar{x})=\{V A+W B:(A, V, W, d) \in \Gamma\},
$$


where $\Gamma$ is the set of all quadruples $(A, B, V, W, d)$ with $A \in$ $T_{f}(\bar{x}), B \in T_{g}(\bar{x}),\|d\|=1, V=\operatorname{diag}\left(v_{i}\right)$, and $W=\operatorname{diag}\left(w_{i}\right)$ being diagonal matrices with

$$
v_{i}=\left\{\begin{aligned}
1- & \frac{f_{i}(\bar{x})^{p-1}}{\left(f_{i}(\bar{x})^{p}+g_{i}(\bar{x})^{p}\right)^{(p-1) / p}}+\alpha g_{i}(\bar{x}), \\
& i \in K(\bar{x}), \\
1- & \frac{\left|A_{i} d\right|^{p-1} \operatorname{sgn}\left(A_{i} d\right)}{\left(\left|A_{i} d\right|^{p}+\left|B_{i} d\right|^{p}\right)^{(p-1) / p},} \\
& i \in J(\bar{x}), \quad\left|A_{i} d\right|^{p}+\left|B_{i} d\right|^{p}>0, \\
1- & \frac{\left|f_{i}(\bar{x})\right|^{p-1} \operatorname{sgn}\left(f_{i}(\bar{x})\right)}{\left(\left|f_{i}(\bar{x})\right|^{p}+\left|g_{i}(\bar{x})\right|^{p}\right)^{(p-1) / p},} \\
& i \notin J(\bar{x}) \cup K(\bar{x}), \\
\operatorname{arbitrary}, & i \in J(\bar{x}), \quad\left|A_{i} d\right|^{p}+\left|B_{i} d\right|^{p}=0,
\end{aligned}\right.
$$$$
w_{i}=\left\{\begin{aligned}
1- & \frac{g_{i}(\bar{x})^{p-1}}{\left(f_{i}(\bar{x})^{p}+g_{i}(\bar{x})^{p}\right)^{(p-1) / p}}+\alpha f_{i}(\bar{x}), \\
& i \in K(\bar{x}), \\
1- & \frac{\left|B_{i} d\right|^{p-1} \operatorname{sgn}\left(B_{i} d\right)}{\left(\left|A_{i} d\right|^{p}+\left|B_{i} d\right|^{p}\right)^{(p-1) / p}}, \\
& i \in J(\bar{x}), \quad\left|A_{i} d\right|^{p}+\left|B_{i} d\right|^{p}>0, \\
1- & \frac{\left|g_{i}(\bar{x})\right|^{p-1} \operatorname{sgn}\left(g_{i}(\bar{x})\right)}{\left(\left|f_{i}(\bar{x})\right|^{p}+\left|g_{i}(\bar{x})\right|^{p}\right)^{(p-1) / p}}, \\
& i \notin J(\bar{x}) \cup K(\bar{x}), \\
\operatorname{arbitrary}, & \\
& i \in J(\bar{x}), \quad\left|A_{i} d\right|^{p}+\left|B_{i} d\right|^{p}=0 .
\end{aligned}\right.
$$

The above calculation relies on the observation that the following is an $H$-differential of the one variable function $\Delta: t \mapsto t_{+}$at any $\bar{t}$ :

$$
\Delta(\bar{t})= \begin{cases}\{1\} & \text { if } \bar{t}>0 \\ \{0,1\} & \text { if } \bar{t}=0 \\ \{0\} & \text { if } \bar{t}<0\end{cases}
$$

Example 6. The following GCP function is based on NCP function in [22]:

$$
\phi_{2}(a, b):=\phi_{p}(a, b)+\alpha(a b)_{+}, \quad \alpha>0 .
$$

We define the GCP function $\Phi(x)$ in (3) as

$$
\Phi_{2}(x):=\phi_{p}(f(x), g(x))+\alpha(f(x) g(x))_{+}, \quad \alpha>0,
$$

where all the operations in (31) are performed componentwise.

Let

$$
\begin{gathered}
J(\bar{x}):=\left\{i: f_{i}(\bar{x})=0=g_{i}(\bar{x})\right\}, \\
L(\bar{x}):=\left\{i: f_{i}(\bar{x}) g_{i}(\bar{x})>0\right\} .
\end{gathered}
$$

Then the $H$-differential of $\Phi_{2}$ at $\bar{x}$ is given by

$$
T_{\Phi_{2}}(\bar{x})=\{V A+W B:(A, V, W, d) \in \Gamma\},
$$

where $\Gamma$ is the set of all quadruples $(A, B, V, W, d)$ with $A \in$ $T_{f}(\bar{x}), B \in T_{g}(\bar{x}),\|d\|=1, V=\operatorname{diag}\left(v_{i}\right)$, and $W=\operatorname{diag}\left(w_{i}\right)$ being diagonal matrices with

$$
\begin{aligned}
& \int 1-\frac{\left|f_{i}(\bar{x})\right|^{p-1} \operatorname{sgn}\left(f_{i}(\bar{x})\right)}{\left(\left|f_{i}(\bar{x})\right|^{p}+\left|g_{i}(\bar{x})\right|^{p}\right)^{(p-1) / p}}+\alpha g_{i}(\bar{x}), \\
& i \in L(\bar{x}) \text {, } \\
& v_{i}=\left\{\begin{array}{c}
1-\frac{\left|A_{i} d\right|^{p-1} \operatorname{sgn}\left(A_{i} d\right)}{\left(\left|A_{i} d\right|^{p}+\left|B_{i} d\right|^{p}\right)^{(p-1) / p}}, \\
i \in J(\bar{x}), \quad\left|A_{i} d\right|^{p}+\left|B_{i} d\right|^{p}>0, \\
1-\frac{\left|f_{i}(\bar{x})\right|^{p-1} \operatorname{sgn}\left(f_{i}(\bar{x})\right)}{\left(\left|f_{i}(\bar{x})\right|^{p}+\left|g_{i}(\bar{x})\right|^{p}\right)^{(p-1) / p}},
\end{array}\right. \\
& i \notin J(\bar{x}) \cup L(\bar{x}) \text {, } \\
& \text { arbitrary, } \\
& i \in J(\bar{x}), \quad\left|A_{i} d\right|^{p}+\left|B_{i} d\right|^{p}=0, \\
& \int 1-\frac{\left|g_{i}(\bar{x})\right|^{p-1} \operatorname{sgn}\left(g_{i}(\bar{x})\right)}{\left(\left|f_{i}(\bar{x})\right|^{p}+\left|g_{i}(\bar{x})\right|^{p}\right)^{(p-1) / p}}+\alpha f_{i}(\bar{x}), \\
& i \in L(\bar{x}) \text {, } \\
& w_{i}=\left\{\begin{aligned}
1- & \frac{\left|B_{i} d\right|^{p-1} \operatorname{sgn}\left(B_{i} d\right)}{\left(\left|A_{i} d\right|^{p}+\left|B_{i} d\right|^{p}\right)^{(p-1) / p}}, \\
& i \in J(\bar{x}), \quad\left|A_{i} d\right|^{p}+\left|B_{i} d\right|^{p}>0, \\
1- & \frac{\left|g_{i}(\bar{x})\right|^{p-1} \operatorname{sgn}\left(g_{i}(\bar{x})\right)}{\left(\left|f_{i}(\bar{x})\right|^{p}+\left|g_{i}(\bar{x})\right|^{p}\right)^{(p-1) / p}},
\end{aligned}\right. \\
& i \notin J(\bar{x}) \cup L(\bar{x}) \text {, } \\
& \text { arbitrary, } \\
& i \in J(\bar{x}), \quad\left|A_{i} d\right|^{p}+\left|B_{i} d\right|^{p}=0 .
\end{aligned}
$$

Example 7. The following GCP function is based on NCP function in [23]:

$$
\phi_{3}(a, b):=\sqrt{\left[\phi_{p}(a, b)\right]^{2}+\alpha\left(a_{+} b_{+}\right)^{2}}, \quad \alpha>0 .
$$

We define the GCP function $\Phi(x)$ in (3) as

$$
\Phi_{3}(x):=\sqrt{\left[\phi_{p}(f(x), g(x))\right]^{2}+\alpha\left(f(x)_{+} g(x)_{+}\right)^{2}}, \quad \alpha>0,
$$

where all the operations in (36) are performed componentwise.

Let

$$
\begin{gathered}
J(\bar{x}):=\left\{i: f_{i}(\bar{x})=0=g_{i}(\bar{x})\right\}, \\
K(\bar{x}):=\left\{i: f_{i}(\bar{x})>0, g_{i}(\bar{x})>0\right\} .
\end{gathered}
$$

When $i \notin K(\bar{x}),\left(\Phi_{3}(\bar{x})\right)_{i}=\left|\left(\Phi_{p}(\bar{x})\right)_{i}\right|=-\left(\Phi_{p}(\bar{x})\right)_{i}$.

Then the $H$-differential of $\Phi_{3}$ at $\bar{x}$ is given by

$$
T_{\Phi_{3}}(\bar{x})=\{V A+W B:(A, V, W, d) \in \Gamma\},
$$

where $\Gamma$ is the set of all quadruples $(A, B, V, W, d)$ with $A \in$ $T_{f}(\bar{x}), B \in T_{g}(\bar{x}),\|d\|=1, V=\operatorname{diag}\left(v_{i}\right)$, and $W=\operatorname{diag}\left(w_{i}\right)$ being diagonal matrices with 


$$
\begin{aligned}
& v_{i}= \begin{cases}\frac{\phi_{p}\left(f_{i}(\bar{x}), g_{i}(\bar{x})\right)\left(1-\left(f_{i}(\bar{x})^{p-1} /\left(f_{i}(\bar{x})^{p}+g_{i}(\bar{x})^{p}\right)^{(p-1) / p}\right)\right)+\alpha f_{i}(\bar{x}) g_{i}^{2}(\bar{x})}{\sqrt{\phi_{p}^{2}\left(f_{i}(\bar{x}), g_{i}(\bar{x})\right)+\alpha\left(f_{i}(\bar{x}), g_{i}(\bar{x})\right)^{2}}}, & i \in K(\bar{x}), \\
\frac{\left|A_{i} d\right|^{p-1} \operatorname{sgn}\left(A_{i} d\right)}{\left(\left|A_{i} d\right|^{p}+\left|B_{i} d\right|^{p}\right)^{(p-1) / p}-1,} & i \in J(\bar{x}), \quad\left|A_{i} d\right|^{p}+\left|B_{i} d\right|^{p}>0,\end{cases} \\
& \frac{\left|f_{i}(\bar{x})\right|^{p-1} \operatorname{sgn}\left(f_{i}(\bar{x})\right)}{\left(\left|f_{i}(\bar{x})\right|^{p}+\left|g_{i}(\bar{x})\right|^{p}\right)^{(p-1) / p}}-1, \quad \quad i \notin J(\bar{x}) \cup K(\bar{x}), \\
& \text { arbitrary, } \\
& i \in J(\bar{x}), \quad\left|A_{i} d\right|^{p}+\left|B_{i} d\right|^{p}=0, \\
& w_{i}= \begin{cases}\frac{\phi_{p}\left(f_{i}(\bar{x}), g_{i}(\bar{x})\right)\left(1-\left(g_{i}(\bar{x})^{p-1} /\left(f_{i}(\bar{x})^{p}+g_{i}(\bar{x})^{p}\right)^{(p-1) / p}\right)\right)+\alpha f_{i}^{2}(\bar{x}) g_{i}(\bar{x})}{\sqrt{\phi_{p}^{2}\left(f_{i}(\bar{x}), g_{i}(\bar{x})\right)+\alpha\left(f_{i}(\bar{x}), g_{i}(\bar{x})\right)^{2}}}, & i \in K(\bar{x}), \\
\frac{\left|B_{i} d\right|^{p-1} \operatorname{sgn}\left(B_{i} d\right)}{\left(\left|A_{i} d\right|^{p}+\left|B_{i} d\right|^{p}\right)^{(p-1) / p}-1,} & i \in J(\bar{x}), \quad\left|A_{i} d\right|^{p}+\left|B_{i} d\right|^{p}>0, \\
\frac{\left|g_{i}(\bar{x})\right|^{p-1} \operatorname{sgn}\left(g_{i}(\bar{x})\right)}{\left(\left|f_{i}(\bar{x})\right|^{p}+\left|g_{i}(\bar{x})\right|^{p}\right)^{(p-1) / p}}-1, & i \notin J(\bar{x}) \cup K(\bar{x}), \\
\text { arbitrary, } & i \in J(\bar{x}), \quad\left|A_{i} d\right|^{p}+\left|B_{i} d\right|^{p}=0 .\end{cases}
\end{aligned}
$$

\section{Minimizing the Merit Function}

In this section, we consider an NCP function $\Phi$ corresponding to GCP and let $\Psi:=(1 / 2)\|\Phi\|^{2}$, when the underlying functions $f$ and $g$ are $H$-differentiable.

It should be recalled that

$$
\Psi(\bar{x})=0 \Longleftrightarrow \Phi(\bar{x})=0 \Longleftrightarrow \bar{x} \text { solves } \operatorname{GCP}(f, g) \text {. }
$$

Let $\Phi$ be an GCP function with an $H$-differential $T_{\Phi}(\bar{x})$ given by

$$
\begin{array}{r}
T_{\Phi}(\bar{x})=\left\{V A+W B: A \in T_{f}(\bar{x}), B \in T_{g}(\bar{x}),\right. \\
\left.V=\operatorname{diag}\left(v_{i}\right), W=\operatorname{diag}\left(w_{i}\right)\right\} .
\end{array}
$$

The following theorem from [15] describes $H$-differential, $T_{\Psi}(\bar{x})$ at $\bar{x}$, for the merit function $\Psi:=(1 / 2)\|\Phi\|^{2}$.

Theorem 8. Suppose $\Phi$ is $H$-differentiable at $\bar{x}$ with $S(\bar{x})$ as an $H$-differential. Then $\Psi:=(1 / 2)\|\Phi\|^{2}$ is $H$-differentiable at $\bar{x}$ with an $H$-differential given by

$$
T_{\Psi}(\bar{x})=\left\{\Phi(\bar{x})^{T} B: B \in S(\bar{x})\right\} .
$$

We need the following Lemma [10] in our subsequent analysis.

Lemma 9. Suppose that $f, g: \mathfrak{R}^{n} \rightarrow \mathfrak{R}^{n}$ and $g$ is one-toone and onto. Define $h: \mathfrak{R}^{n} \rightarrow \mathfrak{R}^{n}$ where $h:=f \circ g^{-1}$. The following hold.

(a) $f$ and $g$ are relatively (strictly) monotone if and only if $h$ is (strictly) monotone.

(b) If $g$ is Lipschitz-continuous and $f$ and $g$ are relatively strongly monotone then $h$ is strongly monotone. (c) $f$ and $g$ are relatively $\mathbf{P}_{\mathbf{0}}(\mathbf{P})$-functions if and only if $h$ is $\mathbf{P}_{\mathbf{0}}(\mathbf{P})$-function.

(d) If $g$ is Lipschitz-continuous and $f$ and $g$ are relatively uniform $\mathbf{P}$-functions, then $h$ is uniform $\mathbf{P}$-function.

The following result is from $[20,24]$.

Theorem 10. Under each of the following conditions, $f$ : $R^{n} \rightarrow R^{n}$ is a $\mathbf{P}_{\mathbf{0}}(\mathbf{P})$-function.

(a) $f$ is Fréchet differentiable on $R^{n}$ and, for every $x \in R^{n}$, the Jacobian matrix $\nabla f(x)$ is a $\mathbf{P}_{\mathbf{0}}(\mathbf{P})$-matrix.

(b) $f$ is locally Lipschitzian on $R^{n}$ and, for every $x \in R^{n}$, the generalized Jacobian $\partial f(x)$ consists of $\mathbf{P}_{\mathbf{0}}(\mathbf{P})$-matrices.

(c) $f$ is semismooth on $R^{n}$ (in particular, piecewise affine or piecewise smooth) and, for every $x \in R^{n}$, the Bouligand subdifferential $\partial_{B} f(x)$ consists of $\mathbf{P}_{\mathbf{0}}(\mathbf{P})$-matrices.

(d) $f$ is $H$-differentiable on $R^{n}$ and, for every $x \in R^{n}$, an $H$-differential $T_{f}(x)$ consists of $\mathbf{P}_{\mathbf{0}}(\mathbf{P})$-matrices.

Remarks. Based on some results in $[20,24]$, we note the following.

(i) For $\mathbf{P}$-conditions, the the converse statements in the above theorem are usually false.

(ii) For $\mathbf{P}_{\mathbf{0}}$-conditions in Theorem 10 , the converse statements of Item (a) and Item (c) are true, while the converse statements of Item (b) and Item (d) may not hold in general $([20,24])$.

It is easy to see the following Lemma. 
Lemma 11. Suppose that $f, g: \mathfrak{R}^{n} \rightarrow \mathfrak{R}^{n}$ and $g$ is one-toone and onto. Define $h: \Re^{n} \rightarrow \Re^{n}$ where $h:=f \circ g^{-1}$. Suppose that $f$ and $g$ are $H$-differentiable at $\bar{x}$ with $H$ differentials, respectively, by $T_{f}(\bar{x})$ and $T_{g}(\bar{x})$ with $T_{g}(\bar{x})$ consisting of nonsingular matrices. Denote $\bar{y}:=\bar{x}$. Then $h$ is $H$-differentiable at $\bar{y}$ with $T_{h}(\bar{y})$, where

$$
T_{h}(\bar{y})=\left\{A B^{-1}: A \in T_{f}(\bar{x}), B \in T_{g}(\bar{x})\right\} .
$$

The following two Lemmas give favorable properties which will be needed in our results.

Lemma 12. We can easily see that $\Phi, V$, and $W$ in Examples 4-6 satisfy the following properties.

(i) $\bar{x}$ solves $G C P(f, g) \Leftrightarrow \Phi(\bar{x})=0$.

(ii) $v_{i}>0, w_{i}>0$, when $\Phi_{i}(\bar{x}) \neq 0$.

(iii) $v_{i}+w_{i} \neq 0$, when $\Phi_{i}(\bar{x}) \neq 0$.

(iv) $\Phi(\bar{x})^{T} v \neq 0$, when $\Phi(\bar{x}) \neq 0$.

(v) $\Phi_{i}(\bar{x})>0$, when $f_{i}(\bar{x})>0, g_{i}(\bar{x})>0$.

Proof. The proof can be easily verified.

Lemma 13. We can easily see that $\Phi, V$, and $W$ in Example 7 satisfy the following properties:

(i) $\bar{x}$ solves $G C P(f, g) \Leftrightarrow \Phi(\bar{x})=0$.

(ii) $v_{i} w_{i}>0$, when $\Phi_{i}(\bar{x}) \neq 0$.

(iii) $v_{i}+w_{i} \neq 0$, when $\Phi_{i}(\bar{x}) \neq 0$.

(iv) $\Phi(\bar{x})^{T} v \neq 0$, when $\Phi(\bar{x}) \neq 0$.

(v) $\Phi_{i}(\bar{x})>0$, when $f_{i}(\bar{x})>0, g_{i}(\bar{x})>0$.

Proof. The proof can be easily verified.

Starting with $H$-differentiable functions $f$ and $g$, we show that, under appropriate conditions, a vector $\bar{x}$ is a solution of the $\operatorname{GCP}(f, g)$ if and only if zero belongs to $T_{\Psi}(\bar{x})$.

In the following theorems we will minimize the merit function under $\mathbf{P}_{\mathbf{0}}(\mathbf{P})$-conditions.

Theorem 14. Suppose $f: \mathfrak{R}^{n} \rightarrow \mathfrak{R}^{n}$ and $g: \mathfrak{R}^{n} \rightarrow \mathfrak{R}^{n}$ are $H$-differentiable at $\bar{x}$ with $H$-differentials, respectively, by $T_{f}(\bar{x})$ and $T_{g}(\bar{x})$. Suppose $\Phi$ is a GCP function of $f$ and $g$. Assume that $\Psi:=(1 / 2)\|\Phi\|^{2}$ is $H$-differentiable at $\bar{x}$ with an $H$-differential given by

$$
\begin{gathered}
T_{\Psi}(\bar{x})=\left\{\Phi(\bar{x})^{T}[V A+W B]: A \in T_{f}(\bar{x}), B \in T_{g}(\bar{x}),\right. \\
V=\operatorname{diag}\left(v_{i}\right), W=\operatorname{diag}\left(w_{i}\right) \\
\text { with } \left.v_{i} w_{i}>0 \text { whenever } \Phi_{i}(\bar{x}) \neq 0\right\} .
\end{gathered}
$$

Further suppose that $T_{g}(\bar{x})$ consists of nonsingular matrices and $S(\bar{x})$ consists of $\mathbf{P}_{0}$-matrices where $S(\bar{x}):=\left\{A B^{-1}: A \in\right.$ $\left.T_{f}(\bar{x}), B \in T_{g}(\bar{x})\right\}$. Then

$$
0 \in T_{\Psi}(\bar{x}) \Longleftrightarrow \Phi(\bar{x})=0 .
$$

Proof. The proof is similar to Theorem 6 in [15].

Remark 15. Theorem 14 is applicable to GCP functions of Examples $4-7$ by the property (ii) in Lemma 12 and the property (ii) in Lemma 13.

A slight modification of the above theorem leads to the following result.

Theorem 16. Suppose $f: \mathfrak{R}^{n} \rightarrow \mathfrak{R}^{n}$ and $g: \mathfrak{R}^{n} \rightarrow \mathfrak{R}^{n}$ are $H$-differentiable at $\bar{x}$ with $H$-differentials, respectively, by $T_{f}(\bar{x})$ and $T_{g}(\bar{x})$. Suppose $\Phi$ is a GCP function of $f$ and $g$. Assume that $\Psi:=(1 / 2)\|\Phi\|^{2}$ is $H$-differentiable at $\bar{x}$ with an $H$-differential given by

$$
\begin{gathered}
T_{\Psi}(\bar{x})=\left\{\Phi(\bar{x})^{T}[V A+W B]: A \in T_{f}(\bar{x}), B \in T_{g}(\bar{x}),\right. \\
V=\operatorname{diag}\left(v_{i}\right), W=\operatorname{diag}\left(w_{i}\right) \\
\text { with } \left.v_{i} w_{i}>0 \text { whenever } \Phi_{i}(\bar{x}) \neq 0\right\} .
\end{gathered}
$$

Further suppose that $T_{g}(\bar{x})$ consists of nonsingular matrices and $S(\bar{x})$ consists of positive semidefinite matrices where $S(\bar{x}):=\left\{A B^{-1}: A \in T_{f}(\bar{x}), B \in T_{g}(\bar{x})\right\}$. Then

$$
0 \in T_{\Psi}(\bar{x}) \Longleftrightarrow \Phi(\bar{x})=0 .
$$

Proof. Since every positive semidefinite matrix is also a $\mathbf{P}_{\mathbf{0}^{-}}$ matrix, the proof follows from Theorem 14.

If $f$ is a monotone (strongly monotone) $C^{1}, \nabla f(\bar{x})$ is positive semidefinite (positive definite) matrix. From Lemma 9, Example 4, and the above theorems, we have the following.

Corollary 17. Suppose $f: R^{n} \rightarrow R^{n}$ and $g: R^{n} \rightarrow$ $R^{n}$ are differentiable at $\bar{x}$. Assume $g$ is continuous and strongly monotone. Moreover, $f$ and $g$ are relatively monotone (relatively strongly monotone) functions. Suppose $\Phi$ is a GCP function of $f$ and $g$, which is based on the generalized FischerBurmeister function and $\Psi:=(1 / 2)\|\Phi\|^{2}$.

Then $\bar{x}$ is a local minimizer to $\Psi$ if and only if $\bar{x}$ solves GCP $(f, g)$.

In view of Example 5 and the above results, we have the following.

Corollary 18. Suppose $f: R^{n} \rightarrow R^{n}$ and $g: R^{n} \rightarrow R^{n}$ are semismooth (piecewise smooth or piecewise affine) at $\bar{x}$ with Bouligand subdifferentials, respectively, by $\partial_{B} f(\bar{x})$ and $\partial_{B} g(\bar{x})$. Assume $g$ is continuous, one-to-one, onto and $\partial_{B} g(\bar{x})$ consists of nonsingular matrices. Moreover, $f$ and $g$ are relatively monotone (relatively strongly monotone) functions. Suppose $\Phi$ is a GCP function of $f$ and $g$, which is based on the generalized Fischer-Burmeister function and $\Psi:=(1 / 2)\|\Phi\|^{2}$.

Then $\bar{x}$ is a local minimizer to $\Psi$ if and only if $\bar{x}$ solves $\operatorname{GCP}(f, g)$.

We state the result for GCP function which is based on the generalized Fischer-Burmeister function. However, as in 
Theorem 14, it is possible to state a very general result for any GCP function $\Phi$ satisfying the properties in Lemmas 12 and 13. For simplicity, we avoid dealing in such a generality.

Corollary 19. Suppose $f: R^{n} \rightarrow R^{n}$ and $g: R^{n} \rightarrow R^{n}$ are differentiable at $\bar{x}$. Suppose $\Phi$ is a GCP function of $f$ and $g$, which is the basis of the generalized Fischer-Burmeister function in Example 4 and $\Psi:=(1 / 2)\|\Phi\|^{2}$. If $\nabla g(\bar{x})$ is nonsingular and the product $\nabla f(\bar{x}) \nabla g(\bar{x})^{-1}$ is $\mathbf{P}_{\mathbf{0}}$-matrix, then $\bar{x}$ is a local minimizer to $\Psi$ if and only if $\bar{x}$ solves $G C P(f, g)$.

Corollary 20. Suppose $f: R^{n} \rightarrow R^{n}$ and $g: R^{n} \rightarrow R^{n}$ are differentiable at $\bar{x}$. Assume $g$ is continuous, one-to-one, onto and $\nabla g(\bar{x})$ is nonsingular. Moreover, assume $f$ and $g$ are relatively $\mathbf{P}_{\mathbf{0}}$-functions. Suppose $\Phi$ is a GCP function of $f$ and $g$, which is based on the generalized square Fischer-Burmeister function in Example 4 and $\Psi:=(1 / 2)\|\Phi\|^{2}$.

Then $\bar{x}$ is a local minimizer to $\Psi$ if and only if $\bar{x}$ solves $G C P(f, g)$.

Proof. Since $g$ is one-to-one and onto and $f$ and $g$ are relatively $\mathbf{P}_{0}$-functions, by Lemma 9 , the mapping $f \circ g^{-1}$ is $\mathbf{P}_{\mathbf{0}}$-function which implies $\nabla f(\bar{x}) \nabla g(\bar{x})^{-1}$ is $\mathbf{P}_{\mathbf{0}}$-matrix; see [20]. The proof follows from Corollary 19.

We recall that a continuous mapping is called a homeomorphism if it is a one-to-one and onto mapping and if its inverse mapping is also continuous.

It is known that a continuous, strongly monotone mapping $f: R^{n} \rightarrow R^{n}$ is a homeomorphism from $R^{n}$ onto itself and the $\nabla f(\bar{x})$ is positive definite matrix if $f$ is $C^{1}$ (see [20]). So we have the following.

Corollary 21. Suppose $f: R^{n} \rightarrow R^{n}$ and $g: R^{n} \rightarrow R^{n}$ are differentiable at $\bar{x}$. Assume $g$ is continuous and strongly monotone. Moreover, assume $f$ and $g$ are relatively $\mathbf{P}_{\mathbf{0}}$-functions. Suppose $\Phi$ is a GCP function of $f$ and $g$, which is based on the generalized Fischer-Burmeister function and $\Psi:=(1 / 2)\|\Phi\|^{2}$. Then $\bar{x}$ is a local minimizer to $\Psi$ if and only if $\bar{x}$ solves $G C P(f, g)$.

Remark 22. When $g(x)=x$ in Corollary 21, we get Proposition 3.4 in [22].

In a view of Example 2, Theorem 3, Corollary 3 in [24], and the above results, we get the following.

Corollary 23. Suppose $f: R^{n} \rightarrow R^{n}$ and $g: R^{n} \rightarrow R^{n}$ are semismooth (piecewise smooth or piecewise affine) at $\bar{x}$ with Bouligand subdifferentials, respectively, by $\partial_{B} f(\bar{x})$ and $\partial_{B} g(\bar{x})$. Assume $g$ is continuous, one-to-one, onto and $\partial_{B} g(\bar{x})$ consists of nonsingular matrices. Moreover, assume $f$ and $g$ are relatively $\mathbf{P}_{0}$-functions. Suppose $\Phi$ is a GCP function of $f$ and $g$, which is based on the generalized Fischer-Burmeister function and $\Psi:=(1 / 2)\|\Phi\|^{2}$.

Then $\bar{x}$ is a local minimizer to $\Psi$ if and only if $\bar{x}$ solves $\operatorname{GCP}(f, g)$.
Theorem 24. Suppose $f: \mathfrak{R}^{n} \rightarrow \mathfrak{R}^{n}$ and $g: \mathfrak{R}^{n} \rightarrow \mathfrak{R}^{n}$ are $H$-differentiable at $\bar{x}$ with $H$-differentials, respectively, by $T_{f}(\bar{x})$ and $T_{g}(\bar{x})$. Suppose $\Phi$ is a GCP function of $f$ and $g$. Assume that $\Psi:=(1 / 2)\|\Phi\|^{2}$ is $H$-differentiable at $\bar{x}$ with an $H$-differential given by

$$
\begin{gathered}
T_{\Psi}(\bar{x})=\left\{\Phi(\bar{x})^{T}[V A+W B]: A \in T_{f}(\bar{x}), B \in T_{g}(\bar{x}),\right. \\
V=\operatorname{diag}\left(v_{i}\right), W=\operatorname{diag}\left(w_{i}\right) \\
\text { with } \Phi^{T}(\bar{x}) v \neq 0 \text { when } \Phi(\bar{x}) \neq 0, \\
\left.v_{i} w_{i} \geq 0 \text { whenever } \Phi_{i}(\bar{x}) \neq 0\right\},
\end{gathered}
$$

or

$$
\begin{gathered}
T_{\Psi}(\bar{x})=\left\{\Phi(\bar{x})^{T}[V A+W B]: A \in T_{f}(\bar{x}), B \in T_{g}(\bar{x}),\right. \\
V=\operatorname{diag}\left(v_{i}\right), W=\operatorname{diag}\left(w_{i}\right) \\
\text { with } v_{i} w_{i} \geq 0, v_{i}+w_{i} \neq 0 \\
\text { whenever } \left.\Phi_{i}(\bar{x}) \neq 0\right\} .
\end{gathered}
$$

Further suppose that $T_{g}(\bar{x})$ consists of nonsingular matrices and $S(\bar{x})$ consists of $\mathbf{P}$-matrices where $S(\bar{x}):=\left\{A B^{-1}: A \in\right.$ $\left.T_{f}(\bar{x}), B \in T_{g}(\bar{x})\right\}$. Then

$$
0 \in T_{\Psi}(\bar{x}) \Longleftrightarrow \Phi(\bar{x})=0 .
$$

Proof. The proof is similar to that of Theorem 9 in [15].

Remark 25. Theorem 24 is applicable to GCP functions of Examples $4-7$ by the properties (i) and (iii) (or (i) and (iv)) in Lemma 12 and (i) and (iii) (or (i) and (iv)) in Lemma 13.

Since every positive definite matrix is also a $\mathbf{P}$-matrix, now we minimize the merit function under positive semidefinite/definite conditions.

Theorem 26. Suppose $f: \mathfrak{R}^{n} \rightarrow \mathfrak{R}^{n}$ and $g: \mathfrak{R}^{n} \rightarrow \mathfrak{R}^{n}$ are $H$-differentiable at $\bar{x}$ with $H$-differentials, respectively, by $T_{f}(\bar{x})$ and $T_{g}(\bar{x})$. Suppose $\Phi$ is a GCP function of $f$ and $g$. Assume that $\Psi:=(1 / 2)\|\Phi\|^{2}$ is $H$-differentiable at $\bar{x}$ with an $H$-differential given by

$$
\begin{gathered}
T_{\Psi}(\bar{x})=\left\{\Phi(\bar{x})^{T}[V A+W B]: A \in T_{f}(\bar{x}), B \in T_{g}(\bar{x}),\right. \\
V=\operatorname{diag}\left(v_{i}\right), W=\operatorname{diag}\left(w_{i}\right) \\
\text { with } \Phi^{T}(\bar{x}) v \neq 0 \text { when } \Phi(\bar{x}) \neq 0, \\
\left.v_{i} w_{i} \geq 0 \text { whenever } \Phi_{i}(\bar{x}) \neq 0\right\},
\end{gathered}
$$

or

$$
\begin{gathered}
T_{\Psi}(\bar{x})=\left\{\Phi(\bar{x})^{T}[V A+W B]: A \in T_{f}(\bar{x}), B \in T_{g}(\bar{x}),\right. \\
V=\operatorname{diag}\left(v_{i}\right), W=\operatorname{diag}\left(w_{i}\right)
\end{gathered}
$$




$$
\begin{aligned}
& \text { with } v_{i} w_{i} \geq 0, v_{i}+w_{i} \neq 0 \\
& \text { whenever } \left.\Phi_{i}(\bar{x}) \neq 0\right\} .
\end{aligned}
$$

Further suppose that $T_{g}(\bar{x})$ consists of nonsingular matrices and $S(\bar{x})$ consists of positive definite matrices where $S(\bar{x}):=$ $\left\{A B^{-1}: A \in T_{f}(\bar{x}), B \in T_{g}(\bar{x})\right\}$. Then

$$
0 \in T_{\Psi}(\bar{x}) \Longleftrightarrow \Phi(\bar{x})=0 .
$$

Now we replace the condition $0 \in T_{\Psi}(\bar{x})$ by weaker conditions $0 \in \operatorname{co} T_{\Psi}(\bar{x})$ or $0 \in \overline{\operatorname{co}} T_{\Psi}(\bar{x})$. In the next two successive theorems, of course, stronger/different conditions on the $H$-differentials of $f$ and $g$ will be imposed. First, we have the following definition.

Remark 27. As noted in [15], a stationary point of the problem $\min f(x)$ is a point $x^{*}$ such that $0 \in \overline{\operatorname{co}} T_{f}\left(x^{*}\right)$ where $T_{f}\left(x^{*}\right)$ is an $H$-differential of $f$ at $x^{*}$. By weakening this condition, we may call a point $x^{*}$ a quasi-stationary point (semistationary point) of the problem $\min f(x)$ if $0 \in T_{f}\left(x^{*}\right)$ (resp., $0 \in \cot T_{f}\left(x^{*}\right)$ ). While local/global minimizers of $\min f(x)$ are stationary points, it is not clear how to get or describe semi- and quasi-stationary points.

We will show that under appropriate conditions when $x^{*}$ is a semistationary point of $\min \Psi$ with $\Psi:=(1 / 2)\|\Phi\|^{2}$, then $x^{*}$ is a solution of a generalized complementarity problem. That is, starting with $H$-differentiable functions $f$ and $g$, we show that under appropriate conditions, a vector $x^{*}$ is a solution of the $\operatorname{GCP}(f, g)$ if and only if zero belongs to $\cot T_{\Psi}\left(x^{*}\right)$.

Definition 28. Consider a nonempty set $\mathscr{C}$ in $\mathfrak{R}^{n \times n}$. We say that a matrix $A$ is a row representative of $\mathscr{C}$ if for each index $i=1,2, \ldots, n$, the $i$ th row of $A$ is the $i$ th row of some matrix $C \in \mathscr{C}$. We say that $\mathscr{C}$ has the row- $\mathbf{P}_{0}$-property (row-Pproperty) if every row representative of $\mathscr{C}$ is a $\mathbf{P}_{\mathbf{0}}$-matrix $(\mathbf{P}$ matrix). We say that $\mathscr{C}$ has the column- $\mathbf{P}_{\mathbf{0}}$-property (column$\mathbf{P}$-property) if $\mathscr{C}^{T}=\left\{A^{T}: A \in \mathscr{C}\right\}$ has the row-P $\mathbf{P}_{\mathbf{0}}$-property (row-P-property).

We have the result from [9].

Proposition 29. A set $\mathscr{C}$ has the row- $\mathbf{P}_{\mathbf{0}}$-property (row-Pproperty) if and only if for each nonzero $x \in \mathfrak{R}^{n}$, there is an index $i$ such that $x_{i} \neq 0$ and $x_{i}(C x)_{i} \geq 0(>0)$ for all $C \in \mathscr{C}$.

A simple consequence of this proposition is the following result in [15].

Proposition 30. The following statements hold.

(i) Suppose the set of matrices $\left\{A^{1}, A^{2}, \ldots, A^{L}\right\}$ has the row- $\mathbf{P}_{\mathbf{0}}$-property. Then for any collection $\left\{V^{1}, V^{2}, \ldots\right.$, $\left.V^{L}\right\}$ of nonnegative diagonal matrices, the sum

$$
A^{*}=\sum_{j=1}^{L} V^{j} A^{j}
$$

is a $\mathbf{P}_{\mathbf{0}}$-matrix. In particular, any convex combination of the $A^{i}$ s is a $\mathbf{P}_{\mathbf{0}}$-matrix.

(ii) Suppose the set of matrices $\left\{A^{1}, A^{2}, \ldots, A^{L}\right\}$ has the row-P-property. Then for any collection $\left\{Y^{1}, \ldots, Y^{L}\right.$, $\left.Z^{*}\right\}$ of nonnegative diagonal matrices with $Y^{1}+\cdots+$ $Y^{L}+Z^{*}>0$

$$
A^{*}=\sum_{j=1}^{L} Y^{j} A^{j}+Z^{*}
$$

is a $\mathbf{P}$-matrix.

Theorem 31. Suppose $f: \mathfrak{R}^{n} \rightarrow \mathfrak{R}^{n}$ and $g: \mathfrak{R}^{n} \rightarrow \mathfrak{R}^{n}$ are $H$-differentiable at $\bar{x}$ with $H$-differentials, respectively, by $T_{f}(\bar{x})$ and $T_{g}(\bar{x})$. Suppose $\Phi$ is a GCP function of $f$ and $g$. Assume that $\Psi:=(1 / 2)\|\Phi\|^{2}$ is $H$-differentiable at $\bar{x}$ with an $H$-differential given by

$$
\begin{gathered}
T_{\Psi}(\bar{x})=\left\{\Phi(\bar{x})^{T}[V A+W B]: A \in T_{f}(\bar{x}), B \in T_{g}(\bar{x}),\right. \\
V=\operatorname{diag}\left(v_{i}\right), W=\operatorname{diag}\left(w_{i}\right) \\
\text { with } \left.v_{i} \geq 0, w_{i}>0 \text { whenever } \Phi_{i}(\bar{x}) \neq 0\right\} .
\end{gathered}
$$

Further suppose $S(\bar{x})$ has the column-P-property where $S(\bar{x}):=$ $T_{f}(\bar{x}) \cup T_{g}(\bar{x})$. Then

$$
0 \in \operatorname{co} T_{\Psi}(\bar{x}) \Longleftrightarrow \Phi(\bar{x})=0 .
$$

Proof. Suppose $\Phi(\bar{x})=0$; then co $T_{\Psi}(\bar{x})=\{0\}$. Conversely, suppose $0 \in \operatorname{co} T_{\Psi}(\bar{x})$. Then by Carathéodory's theorem, there exist $\Phi(\bar{x})^{T}\left[V^{j} A^{j}+W^{j} B^{j}\right] \in T_{\Psi}(\bar{x})$ and scalars $\lambda_{j}>0$, $A^{j} \in T_{f}(\bar{x})$, and $B^{j} \in T_{g}(\bar{x})$ for $j=1,2, \ldots, L$ with $L \leq n+1$ such that

$$
\sum_{j=1}^{L} \lambda_{j} \Phi(\bar{x})^{T}\left[V^{j} A^{j}+W^{j} B^{j}\right]=0
$$

where $\sum_{j=1}^{L} \lambda_{j}=1$. Then (58) can be rewritten as

$$
\Phi(\bar{x})^{T}\left[Y^{1} A^{1}+\cdots+Y^{L} A^{L}+Z^{1} B^{1}+\cdots+Z^{L} B^{L}\right]=0,
$$

where $\lambda_{j} V^{j}=Y^{j}$ and $\lambda_{j} W^{j}=Z^{j}$ for all $j$. And (59) can reduce to

$$
\left(C^{*}\right)^{T} \Phi(\bar{x})=0
$$

where $C^{*}=Y^{1} A^{1}+\cdots+Y^{L} A^{L}+Z^{1} B^{1}+\cdots+Z^{L} B^{L}$. From (60), we have

$$
\left[\left(C^{*}\right)^{T} \Phi(\bar{x})\right]_{i}=0, \quad \text { for } i=1,2, \ldots, n .
$$

Then

$$
\Phi_{i}(\bar{x})\left[\left(C^{*}\right)^{T} \Phi(\bar{x})\right]_{i}=0, \quad \text { for } i=1,2, \ldots, n .
$$


Now, we claim that $\Phi(\bar{x})=0$. Assume the contrary that $\Phi(\bar{x}) \neq 0$; then for $\Phi(\bar{x})$, from Proposition 29, there is an index $i_{0}$ such that $\Phi_{i_{0}}(\bar{x}) \neq 0$ and

$$
\Phi_{i_{0}}(\bar{x})\left[C^{T} \Phi(\bar{x})\right]_{i_{0}}>0, \quad \forall C \in\left\{A^{1}, \ldots, A^{L}, B^{1}, \ldots, B^{L}\right\} .
$$

Note that $v_{i_{0}}^{j} \geq 0, w_{i_{0}}^{j}>0$ and $\lambda_{j}>0$ for $j=1,2, \ldots, L$; then we have

$$
\begin{aligned}
& \Phi_{i_{0}}(\bar{x})\left[\left(Y^{j} A^{j}\right)^{T} \Phi(\bar{x})\right]_{i_{0}}=\lambda_{j} v_{i_{0}}^{j} \Phi_{i_{0}}(\bar{x})\left[\left(A^{j}\right)^{T} \Phi(\bar{x})\right]_{i_{0}} \geq 0, \\
& \Phi_{i_{0}}(\bar{x})\left[\left(Z^{j} B^{j}\right)^{T} \Phi(\bar{x})\right]_{i_{0}}=\lambda_{j} w_{i_{0}}^{j} \Phi_{i_{0}}(\bar{x})\left[\left(B^{j}\right)^{T} \Phi(\bar{x})\right]_{i_{0}}>0,
\end{aligned}
$$

for $j=1,2, \ldots, L$. Then we obtain

$$
\begin{aligned}
\Phi_{i_{0}}(\bar{x})\left[\left(C^{*}\right)^{T} \Phi(\bar{x})\right]_{i_{0}}= & \sum_{j=1}^{L} \Phi_{i_{0}}(\bar{x})\left[\left(Y^{j} A^{j}\right)^{T} \Phi(\bar{x})\right]_{i_{0}} \\
& +\sum_{j=1}^{L} \Phi_{i_{0}}(\bar{x})\left[\left(Z^{j} B^{j}\right)^{T} \Phi(\bar{x})\right]_{i_{0}}>0 .
\end{aligned}
$$

(65) contradicts $(62)$. This proves that $\Phi(\bar{x})=0$.

Remark 32. (i) When $g(x)=x$, the GCP reduces to NCP.

(ii) Theorem 31 is applicable to GCP functions of Examples $4-7$ by the properties (ii) in Lemma 12 and (ii) in Lemma 13.

Concluding Remarks. We considered a generalized complementarity problem corresponding to $H$-differentiable functions, with an associated GCP function $\Phi$ and a merit function $\Psi(x)=(1 / 2)\|\Phi\|^{2}$. In this paper, we showed under certain $\mathbf{P}\left(\mathbf{P}_{\mathbf{0}}\right)$-conditions the global/local minimum or a stationary point of $\Psi$ is a solution of $\operatorname{GCP}(f, g)$. For generalized complementarity problem based on the penalized Fischer-Burmeister function, our results give various results for generalized complementarity problem when the underlying functions are continuously differentiable (locally Lipschitzian, semismooth, and directionally differentiable) functions. For example, we have the following.

(i) When $g(x)=x$ in this paper, then $\mathrm{GCP}(f, g)$ reduces to nonlinear complementarity problem $\mathrm{NCP}(f)$ and we get results for nonsmooth $\operatorname{NCP}(f)$ based on the generalized Fischer-Burmeister function and its generalizations which seems to be new.

(ii) When $f$ and $g$ are $C^{1}$ in which case $T_{f}(\bar{x})=\{\nabla f(\bar{x})\}$ and $T_{g}(\bar{x})=\{\nabla g(\bar{x})\}$, our results will be true when the underlying functions are $C^{1}$ and get the results in [25].

(iii) When $f$ is $C^{1}$ and $g(x)=x$ (in which case we can let $\left.T_{f}(\bar{x})=\{\nabla f(\bar{x})\}\right), \operatorname{GCP}(f, g)$ reduces to nonlinear complementarity problem $\mathrm{NCP}(f)$, and the results of this paper will be valid for $\operatorname{NCP}(f)$. (iv) When $f$ is locally Lipschitzian with $T_{f}(\bar{x})=\partial f(\bar{x})$ and $g(x)=x$, our results will be applicable to $\mathrm{NCP}(f)$ when the underlying data are locally Lipschitzian.

(v) Our results give various results for generalized complementarity problem when $p$-norm is replaced by 2 norm (or when $p$ is an integer greater than 2), we can state our results for GCP function based on the Fischer-Burmeister function and we get the following.

(a) When $f$ and $g$ are $C^{1}$ in which case $T_{f}(\bar{x})=$ $\{\nabla f(\bar{x})\}$ and $T_{g}(\bar{x})=\{\nabla g(\bar{x})\}$, Theorem 14 reduces to Theorem 3.2 in [26].

(b) When $f$ is $C^{1}$ and $g(x)=x$ (in which case we can let $\left.T_{f}(\bar{x})=\{\nabla f(\bar{x})\}\right), \operatorname{GCP}(f, g)$ reduces to $\operatorname{NCP}(f)$ and the above result reduces to Prop. 3.4 in [27].

(c) When $f$ is locally Lipschitzian with $T_{f}(\bar{x})=$ $\partial f(\bar{x})$ and $g(x)=x$, the above theorem reduces to a result for nonlinear complementarity problem by Fischer [28]. Moreover, when $g(x)=x$, our result extends/generalizes a result obtained by Geiger and Kanzow [29] for $\operatorname{NCP}(f)$ under monotonicity of a $C^{1}$ function and by Jiang [30] under uniform $\mathbf{P}$-property of a directionally differentiable function.

To the best of our knowledge, solving nonsmooth $\operatorname{GCP}(f, g)$ on the basis of the generalized Fischer-Burmeister function and its generalizations seems to be new.

\section{Conflict of Interests}

The authors declare that there is no conflict of interests regarding the publication of this paper.

\section{Acknowledgments}

The research of Mohamed A. Tawhid is supported in part by the Natural Sciences and Engineering Research Council of Canada (NSERC). The postdoctoral fellowship of Wei-Zhe $\mathrm{Gu}$ is supported by NSERC. The research of Wei-Zhe $\mathrm{Gu}$ is supported in part by the National Natural Science foundation of China (Grant no. 11301375; Grant no. 71301118), Research Fund for the Doctoral Program of Higher Education of China (Grant no. 20120032120076), and Tianjin Planing Program of Philosophy and Social Science (Grant no. TJTJ11-004).

\section{References}

[1] M. S. Gowda and G. Ravindran, "Algebraic univalence theorems for nonsmooth functions," Journal of Mathematical Analysis and Applications, vol. 252, no. 2, pp. 917-935, 2000.

[2] F. H. Clarke, Optimization and Nonsmooth Analysis, SIAM, Philadelphia, Pa, USA, 1990.

[3] R. Mifflin, "Semismooth and semiconvex functions in constrained optimization," SIAM Journal on Control and Optimization, vol. 15, no. 6, pp. 959-972, 1977. 
[4] L. Q. Qi, "Convergence analysis of some algorithms for solving nonsmooth equations," Mathematics of Operations Research, vol. 18, no. 1, pp. 227-244, 1993.

[5] L. Q. Qi and J. Sun, "A nonsmooth version of Newton's method," Mathematical Programming, vol. 58, no. 3, pp. 353-367, 1993.

[6] L. Qi, C-Differential Operators, C-Differentiability and Generalized Newton Methods, Research Report, School of Mathematics, The University of New South Wales, Sydney, Australia, 1996.

[7] M. A. Tawhid, "On the local uniqueness of solutions of variational inequalities under $H$-differentiability," Journal of Optimization Theory and Applications, vol. 113, no. 1, pp. 149-164, 2002.

[8] M. S. Gowda, "Inverse and Implicit Function Theorems for H-Differentiable and Semismooth Functions," Optimization Methods \& Software, vol. 19, no. 5, pp. 443-461, 2004.

[9] Y. Song, M. S. Gowda, and G. Ravindran, "On some properties of P-matrix sets," Linear Algebra and Its Applications, vol. 290, no. 1-3, pp. 237-246, 1999.

[10] M. A. Tawhid, "An application of H-differentiability to nonnegative and unrestricted generalized complementarity," Computational Optimization and Applications, vol. 39, no. 1, pp. 5174, 2008.

[11] M. A. Tawhid, "Solution of nonsmooth generalized complementarity problems," Journal of the Operations Research Society of Japan, vol. 54, no. 1, pp. 12-24, 2011.

[12] M. A. Tawhid, "Unconstrained methods for nonsmooth nonlinear complementarity problems," Advanced Modeling and Optimization, vol. 12, no. 1, pp. 20-35, 2010.

[13] M. A. Tawhid, "Nonsmooth generalized complementarity as unconstrained optimization," Journal of Industrial and Management Optimization, vol. 6, no. 2, pp. 411-423, 2010.

[14] M. A. Tawhid and J. L. Goffin, "On Minimizin g Some Merit Functions for Non-linear Complementarity Problems under $\mathrm{H}$ differentiability," Journal of Optimization Theory and Applications, vol. 139, no. 1, pp. 127-140, 2008.

[15] M. A. Tawhid and M. S. Gowda, "On two applications of Hdifferentiability to optimization and complementarity problems," Computational Optimization and Applications, vol. 17, no. 2-3, pp. 279-299, 2000.

[16] D. H. Hyers, G. Isac, and T. M. Rassias, Topics in Nonlinear Analysis \& Applications, World Scientific, Singapore, 1997.

[17] G. Isac, Complementarity Problems, vol. 1528 of Lecture Notes in Mathematics, Springer, Berlin, Germany, 1992.

[18] M. A. Noor, "The quasi-complementarity problem," Journal of Mathematical Analysis and Applications, vol. 130, no. 2, pp. 344353, 1988.

[19] J. S. Pang, “The implicit complementarity problem," in Nonlinear Programming, O. L. Mangasarian, R. R. Meyer, and S. M. Robinson, Eds., pp. 487-518, Academic Press, New York, NY, USA, 1981.

[20] J. J. Moré and W. C. Rheinboldt, "On P- and S-functions and related classes of n-dimensional nonlinear mappings," Linear Algebra and Its Applications, vol. 6, pp. 45-68, 1973.

[21] P. Tseng, "Growth behavior of a class of merit functions for the nonlinear complementarity problem," Journal of Optimization Theory and Applications, vol. 89, no. 1, pp. 17-37, 1996.

[22] J.-S. Chen and S.-H. Pan, "A family of NCP functions and a descent method for the nonlinear complementarity problem," Computational Optimization and Applications, vol. 40, no. 3, pp. 389-404, 2008.
[23] J. Chen, "On some NCP-functions based on the generalized Fischer-Burmeister function," Asia-Pacific Journal of Operational Research, vol. 24, no. 3, pp. 401-420, 2007.

[24] Y. Song, M. S. Gowda, and G. Ravindran, "On characterizations of P- and $P_{0}$-properties in nonsmooth functions," Mathematics of Operations Research, vol. 25, pp. 400-408, 2000.

[25] G. Wei-Zhe and M. A. Tawhid, "Generalized complementarity problems based on generalized Fisher-Burmeister functions as unconstrained optimization," Advanced Modeling and Optimization, vol. 16, no. 2, pp. 269-284, 2014.

[26] C. Kanzow and M. Fukushima, "Equivalence of the generalized complementarity problem to differentiable unconstrained minimization," Journal of Optimization Theory and Applications, vol. 90, no. 3, pp. 581-603, 1996.

[27] F. Facchinei and J. Soares, "A new merit function for nonlinear complementarity problems and a related algorithm," SIAM Journal on Optimization, vol. 7, no. 1, pp. 225-247, 1997.

[28] A. Fischer, "Solution of monotone complementarity problems with locally Lipschitzian functions," Mathematical Programming, vol. 76, no. 3, Ser. B, pp. 513-532, 1997.

[29] C. Geiger and C. Kanzow, "On the resolution of monotone complementarity problems," Computational Optimization and Applications, vol. 5, no. 2, pp. 155-173, 1996.

[30] H. Jiang, "Unconstrained minimization approaches to nonlinear complementarity problems," Journal of Global Optimization, vol. 9, no. 2, pp. 169-181, 1996. 


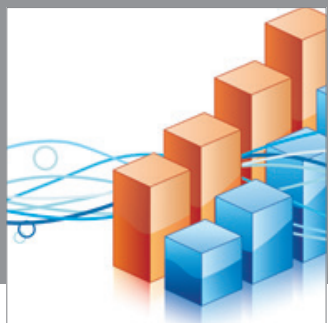

Advances in

Operations Research

mansans

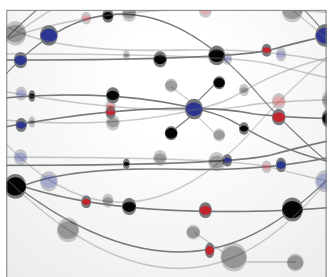

The Scientific World Journal
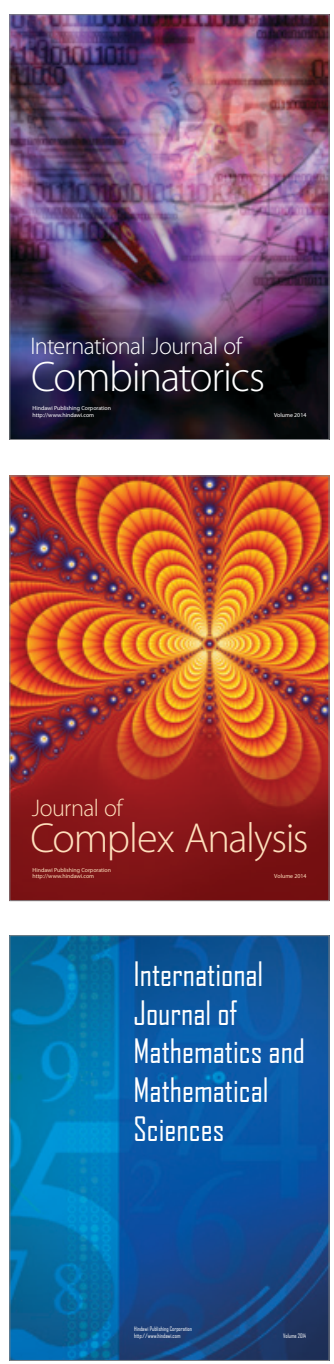
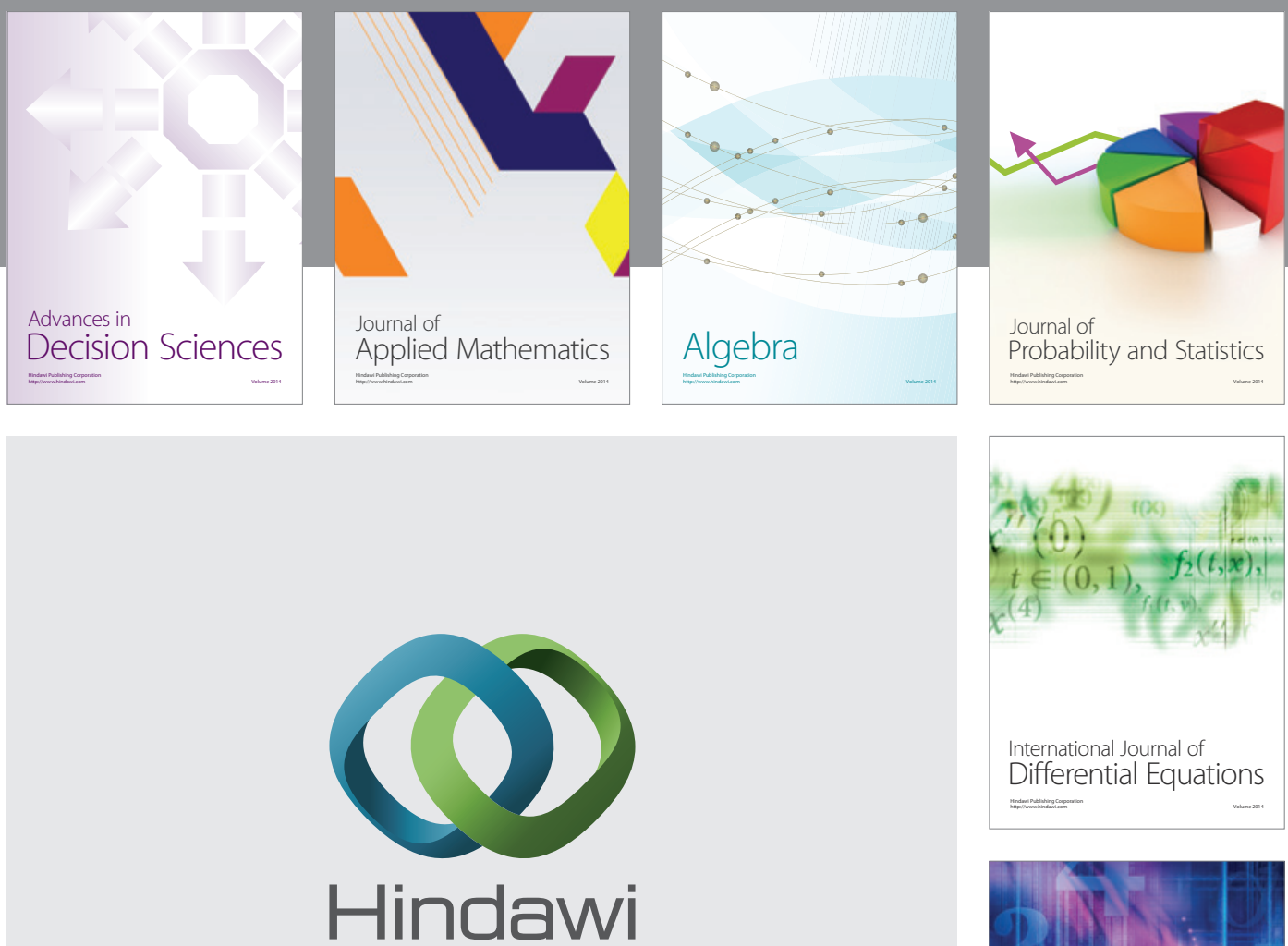

Submit your manuscripts at http://www.hindawi.com
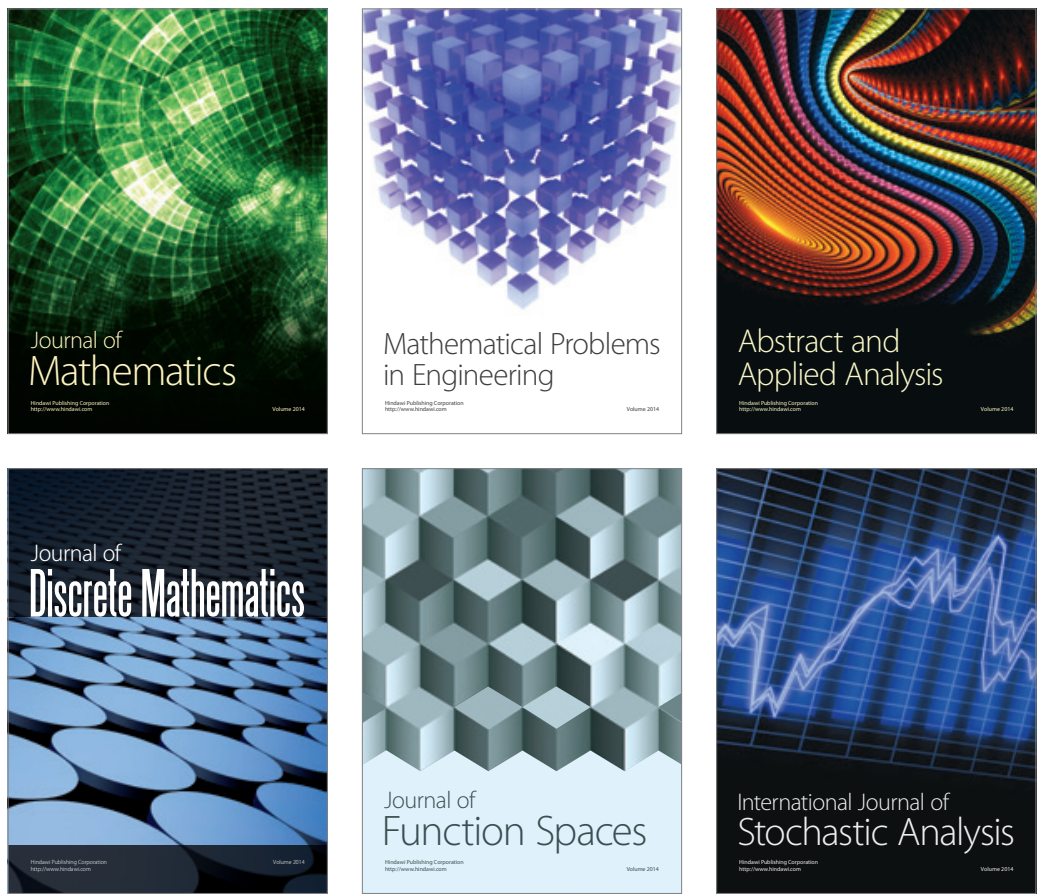

Journal of

Function Spaces

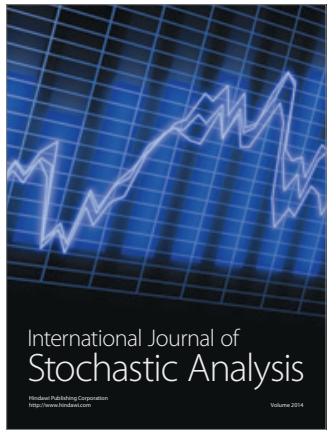

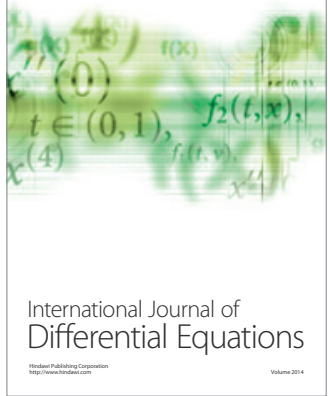
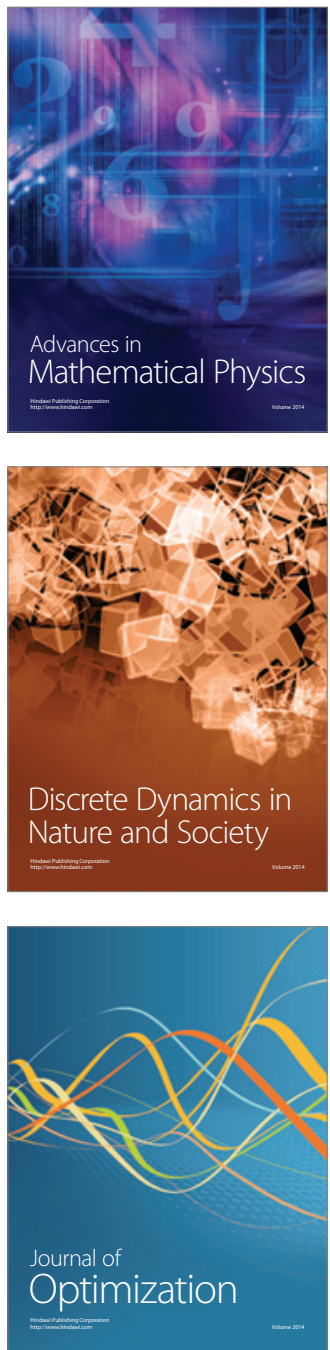